\title{
Adaptive Nonparametric Control Chart for Time-Varying and Multimodal Processes
}

\author{
Ji Hoon Kang', Jaehong Yu², Seoung Bum Kim²* \\ ${ }^{1}$ Industrial Technology Laboratory, Algorithm Research Center \\ Samsung SDS \\ Sincheon-dong, Songpa-gu, Seoul 138-240 \\ Republic of Korea \\ ${ }^{2}$ Department of Industrial Management Engineering \\ Korea University \\ Anam-dong, Seongbuk-gu, Seoul 136-713 \\ Republic of Korea \\ sbkim1@,korea.ac.kr \\ Tel. 82-2-3290-3397, 82-2-3290-3397
}

Fax 82-2-929-5888

* Corresponding author 


\title{
Adaptive Nonparametric Control Chart for Time-Varying and Multimodal Processes
}

\begin{abstract}
Multivariate statistical process control techniques have been widely used to improve processes by reducing variation and preventing defects. In modern manufacturing, because of the complexity and variability of processes, traditional multivariate control charts such as Hotelling's $\mathrm{T}^{2}$ cannot efficiently handle situations in which the patterns of process observations are nonlinear, multimodal, and time varying. In the present study, we propose a nonparametric control chart, which is capable of adaptively monitoring time-varying and multimodal processes. Experiments with simulated and real process data from a thin film transistor-liquid crystal display (TFT-LCD) demonstrate the effectiveness and accuracy of the proposed method.
\end{abstract}

Keywords: clustering, data mining algorithm, multivariate control chart; multimodality; timevarying process; false alarms 


\section{Introduction}

Statistical process control (SPC) is a practical and effective method for diagnosing problems in manufacturing processes and preventing defective products. The main advantage of SPC is to minimize the variability of a process by detecting assignable causes that permit immediate remedial actions (Woodall, 2000; Mason et al. 1997). A control chart is a representative SPC technique that monitors process stability over time to maintain a process in a normal state. Because their construction is based on solid statistical theory, control charts have been widely used. Furthermore, control charts provide a comprehensive graphical output that engineers can easily configure with minimal assistance (Montgomery, 2005).

Shewhart (1939) suggested the use of a univariate control chart to monitor a single quality characteristic. One that is widely used is a $\bar{x}$ control chart. The monitoring statistics in a $\bar{x}$ control chart can be calculated by taking an average of each sample or subgroup. Control limits can be determined by setting $\alpha$, which is based upon the probability distribution. However, in practice, univariate control charts have some limitations because of their inability to reflect relationships between the variables in multivariate processes. Modern manufacturing systems usually contain a variety of correlated quality characteristics. Thus, unregulated use of univariate control charts in multivariate processes may lead to unsatisfactory conclusions (e.g., an increase in false alarms) (Phaladiganon et al., 2013; Bersimis et al., 2006).

Because of the limitations of univariate charts, multivariate statistical process control methods that can simultaneously encompass information on multiple quality characteristics have been introduced to detect and prevent product defects in modern manufacturing systems (Runger, 1996; Woodall, 2000). The most well-known and widely used is a Hotelling's $\mathrm{T}^{2}$ control chart. 
The monitoring statistic of a $\mathrm{T}^{2}$ chart is the distance between an observation and the center of incontrol observations in multidimensional space. This distance is defined by the mean vectors of the quality characteristics and their covariance matrix. The control limit of a $\mathrm{T}^{2}$ control chart is proportional to the percentile of the F-distribution (Montgomery, 2005). Although traditional $\mathrm{T}^{2}$ control charts are still widely used as a representative quality control method, they have some parametric restrictions. One is that in-control observations should follow the multivariate normal distribution, which may not be always true in real manufacturing systems (Chakraborti et al., 2001).

To overcome this shortcoming, some nonparametric multivariate control charts have been suggested. Liu and Singh (1993) proposed a nonparametric $r$ chart based on ranking depth. However, a ranking depth chart may fail to detect some out-of-control observations when the data follow the multivariate normal distribution (Mason et al., 1997). Chou et al. (2001) proposed a nonparametric $\mathrm{T}^{2}$ control chart based on a kernel density estimation technique that can estimate the distribution of $\mathrm{T}^{2}$ statistics and calculate a control limit without any parametric assumptions. However, its operation requires determining several parameters such as the kernel function, smoothing, and the number of spaced points. Alternatively, Phaladiganon et al. (2011) proposed a bootstrap-based approach to establish a control limit when there is no information on the parametric distribution.

Several attempts have been made to combine classification algorithms with multivariate control charts to refine the monitoring statistics (Sukchotrat et al., 2011; Hwang et al., 2007; Chongfuangprinya et al., 2011; Kim et al., 2012). These classification-based control charts can detect out-of-control observations more accurately than traditional multivariate control charts, especially under nonnormal situations. Furthermore, some studies introduced multivariate 
control chart techniques based on one-class classification (OCC) algorithms. They proposed the use of the novelty scores of OCC algorithms as the monitoring statistics of a control chart. OCCbased control charts have performed better than their alternatives in many nonnormal cases (Tuerhong et al., 2014; Kim et al., 2011; Sukchotrat et al., 2010; Sun and Tsung, 2003).

Despite these efforts, most multivariate control charts rely on an assumption that a single distribution generates the in-control observations. However, in current manufacturing systems, process conditions can be readily shifted because of changes in various factors, and thus, they can have multimodal properties (Yu and Qin, 2008).

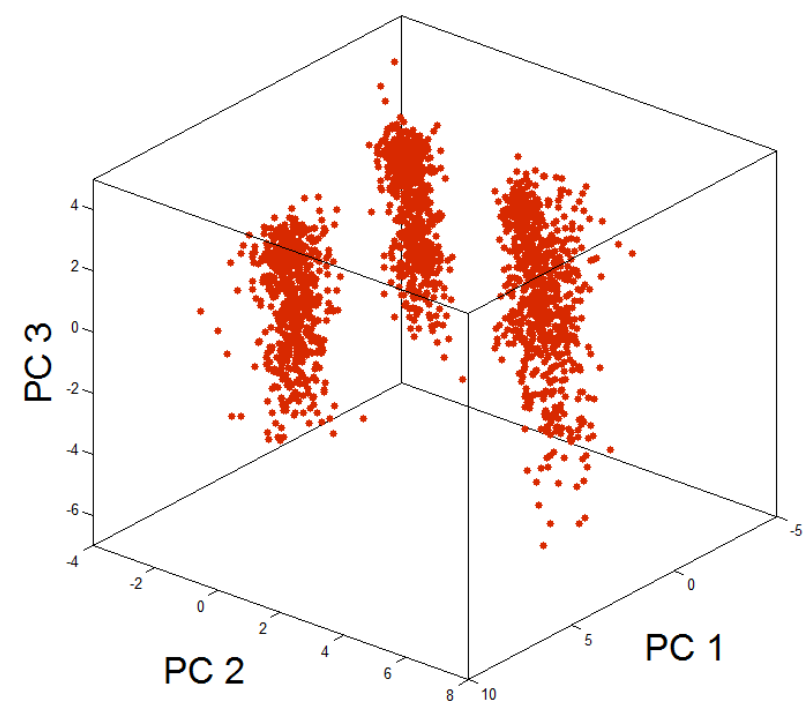

Figure 1. A three-dimensional principal component score plot of in-control observations from a thin film transistor-liquid crystal display process.

Figure 1 shows a three-dimensional principal component score plot showing that in-control observations in the fabrication generated during the manufacturing process for a thin film transistor-liquid crystal display (TFT-LCD) exhibit a multimodal pattern. This example comes 
from the several process chambers of TFT-LCD fabrication in one of the leading display companies in South Korea.

The inefficiency of existing methods in dealing with multimodal processes has motivated the development of several monitoring techniques based on recursive or adaptive principal component analysis and partial least squares algorithms (Wang et al., 2005; Li et al., 2000). These methods integrate feature extraction algorithms and local update strategies to address multimodal problems. However, they are either based on labor-intensive models or too complicated to be used in practice (Ge and Song, 2008). Recently, a Gaussian mixture model (GMM) chart and a clustering-based Mahalanobis (CBM) chart have been proposed to handle multimodal processes efficiently (Kang and Kim, 2013; Yu, 2012; Qin, 2008).

In addition to multimodal problems, a manufacturing process also often involves a timevarying pattern. These patterns are often encountered because of human-caused disturbances such as set-point changes or other unwanted disturbances such as aged equipment, fouling, and catalyst degradation (Choi et al., 2006; Kourti et al., 1995). Time-varying patterns can be considered as an especially critical issue in many electronic and chemical engineering fields ( $\mathrm{Li}$ et al., 2000; Qin, 1998). Ge and Song (2008) proposed an adaptive monitoring scheme in the nonlinear and multimodal processes by applying least square support vector regression (LSSVR). Because of its computational efficiency compared with existing support vector regression, the LSSVR can be adaptively updated to enable online process monitoring. More recently, Xie and Shi (2012) suggested a dynamic modeling method for GMM charts for multimodal processes. They proposed a moving window-based update logic to find the most probable Gaussian component to receive assignment of a new observation. This method dynamically decides through a moving window which components to update and which ones to leave unchanged. 
In the present study, we propose a nonparametric multivariate control chart technique that can simultaneously handle time-varying and multimodal processes. We call the proposed method a time-adaptive clustering-based control chart because it uses a clustering algorithm to handle multimodal situations and also because with a clustering algorithm, it can trace the changes in processes over time and keep the control chart up to date.

The remainder of this study is organized as follows. Section 2 reviews existing monitoring methodologies for time-varying and multimode processes. In particular, we focus on reviewing Gaussian mixture model-based control charts, the latest methodologies. Section 3 describes the proposed TAC-based control chart by emphasizing its online adaptive strategy for time-varying and multimodal processes. Section 4 presents a simulation study to examine the performance of the proposed TAC-based control chart under various scenarios. Section 5 presents a case study using an actual TFT-LCD process that evaluates the performance of the proposed monitoring technique. Finally, Section 6 contains concluding remarks.

\section{Existing monitoring methodologies for time-varying and multimode processes}

Gaussian mixture model (GMM) is widely used to construct group structure assuming that each group follows the Gaussian distribution. More precisely, a GMM model describes an original data with several Gaussian components, and each component is considered as a mode best representing the local distribution (Figueiredo and Jain, 2002). Thus, GMM can appropriately reflect the local properties of a dataset. Several versions of the GMM-based control charts have been proposed to address the multimodality issues found in the modern manufacturing systems (Choi et al., 2004; Yu and Qin, 2008). 
To calculate the monitoring statistic of GMM-based control chart, the posterior probability of monitored observation $x_{t}$ belonging to $i^{\text {th }}$ mode $\left(p\left(x_{t} \in C_{i}\right)\right)$ and the local Mahalonobis distance $\left(D\left(x_{t}, C_{i}\right)\right)$ can be defined as follows:

$$
p\left(x_{t} \in C_{i}\right)=\frac{\alpha_{i} g\left(x_{t} \mid \mu_{i}, \Sigma_{i}\right)}{\sum_{j=1}^{K} \alpha_{j} g\left(x_{t} \mid \mu_{j,}, \Sigma_{j}\right)},
$$

where $\alpha_{i}$ is a prior probability of Gaussian component $i$, and $g\left(x_{t} \mid \mu_{i}, \Sigma_{i}\right)$ is a density function of the Gaussian component $i$ with mean vector $\left(\mu_{i}\right)$ and covariance matrix $\left(\Sigma_{i}\right)$.

$$
D\left(x_{t}, C_{i}\right)=\left(x_{t}-\mu_{i}\right)^{T} \Sigma_{i}^{-1}\left(x_{t}-\mu_{i}\right) .
$$

In Equation (1), the membership of observation $\left(x_{t}\right)$ is expressed as its probability ( $p\left(x_{t} \in\right.$ $\left.C_{i}\right)$ ). In addition, local Mahalanobis distance in Equation (2) indicates the deviation from the mean vector of $i^{\text {th }}$ Gaussian component. Using these two factors, final monitoring statistic of GMM-based control chart, $D_{\text {global }}$, can be computed as follows:

$$
D_{\text {global }}=\sum_{i=1}^{k} p\left(x_{t} \in C_{i}\right) D\left(x_{t}, C_{i}\right)
$$

In Equation (3), a monitoring statistic is combination of the membership $p\left(x_{t} \in C_{i}\right)$ belonging to certain Gaussian component and the local Mahalonobis distance $\left(D\left(x_{t}, C_{i}\right)\right)$. The control limit $(C L)$ is constructed with the percentile of the F-distribution as follows:

$$
C L=\frac{p\left(n^{2}-1\right)}{n(n-p)} F_{p, n-p ; \alpha},
$$

where $n$ and $p$ are, respectively, the number of observations and variables in the in-control observations.

The GMM-based charts have been successfully used to monitor the multimodal processes. However, the performance of the monitoring scheme can be degraded when the natural changes caused by equipment aging and convection are occurred in in-control processes (Xie et al., 
2012)._To address these limitations, Xie et al. (2012) extended the idea of the existing GMMbased monitoring method. In this approach, the mean vector and covariance matrix of the $i^{\text {th }}$ Gaussian component are updated to accommodate the natural changes that exist in in-control processes. The mean vector $\left(\mu_{i}^{(t)}\right)$ and covariance matrix $\left(\Sigma_{i}^{(t)}\right)$ can be updated by the following equations:

$$
\mu_{i}^{(t)}=\mu_{i}^{(t-1)}+\frac{1}{N}\left(x_{t}-x_{t-N}\right) I\left(x_{t} \in C_{i}\right)
$$

where $x_{t}$ is $t^{\text {th }}$ observation, and $N$ is the window size.

$$
\begin{gathered}
\Sigma_{i}^{(t)}=\Sigma_{i}^{(t-1)}+\left(\frac{1}{N-1}\left(\left(x_{t}-\mu_{i}^{(t)}\right)^{T}\left(x_{t}-\mu_{i}^{(t)}\right)-\left(x_{t-N}-\mu_{i}^{(t-N)}\right)^{T}\left(x_{t-N}-\mu_{i}^{(t-N)}\right)\right)+\right. \\
\left.\Delta \mu_{i}^{(t)^{T}} \Delta \mu_{i}^{(t)}-\Delta \mu_{i}^{(t-1)^{T}} \Delta \mu_{i}^{(t-1)}\right) I\left(x_{t} \in C_{i}\right)
\end{gathered}
$$

where $\Delta \mu_{i}^{(t)}=\mu_{i}^{(t)}-\mu_{i}^{(t-1)}$ and $I\left(x_{t} \in C_{i}\right)$ is the indicator function, defined as follows.

$$
I\left(x_{t} \in C_{i}\right)=\left\{\begin{array}{rr}
1, & \text { if } p\left(x_{t} \in C_{i}\right)=\max _{j} p\left(x_{t} \in C_{j}\right) \\
0, & \text { otherwise }
\end{array}\right.
$$

It can be noted that the mean vector and covariance matrix are updated within the moving window to reflect recent status of process. A window size is the weighting parameter that determines the amount of update. For example, if $N$ is small, we give more weights on the current observations than the past observations. However, if $N$ is large, the effect of recent observations may be less. In general, the window size $(N)$ is empirically determined to give the lowest error rate.

Although a time-varying version of the GMM-based chart performs reasonably well, this method still have an assumption that each component of the mixture follows the Gaussian 
distribution. In reality, however, many manufacturing processes exhibit significant nonnormality and nonlinearity in their operations, and thus the GMM-based charts cannot be accommodated for nonnormal and nonlinear patterns (Yu, 2012; Zhang et al., 2013). This motivates the focus of this paper on the development of the control chart technique to handle time-varying and multimodal processes under nonnormal and nonlinear situations.

\section{Proposed methodology: time-adaptive clustering-based control chart}

As described in the Introduction, nonnormal and multimodal characteristics are frequently encountered in manufacturing processes (Kano et al., 2003; Lee et al., 2004). In this situation, existing multivariate monitoring methods applied without any modification may be unsatisfactory because current methods are based on an assumption that the in-control observations are in unimode.

Recently, a clustering-based control chart was proposed to address multimodal processes without assuming any parametric distributions of the data (Kang and Kim, 2013). A clustering analysis systematically partitions observations by minimizing within-group variations and maximizing between-group variations and then assigns a cluster label to each observation (Zhang and Rudnicky, 2002). Figure 2 (a) shows the $\mathrm{T}^{2}$ control boundary generated by in-control observations, and Figure 2 (b) shows five $\mathrm{T}^{2}$ control boundaries after a $k$-means clustering algorithm $(k=5)$ was used to cluster in-control observations into five groups. We learned from this example that partitioning the samples into smaller subgroups can better represent the structure of nonlinear and multimodal processes. This example clearly shows that each subgroup 
formed by a clustering algorithm can be closer to the single Gaussian distribution than the original in-control observations.

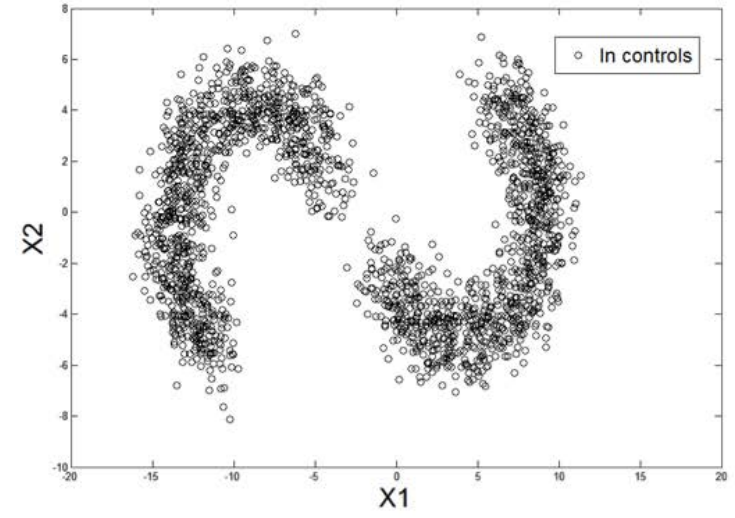

(a)

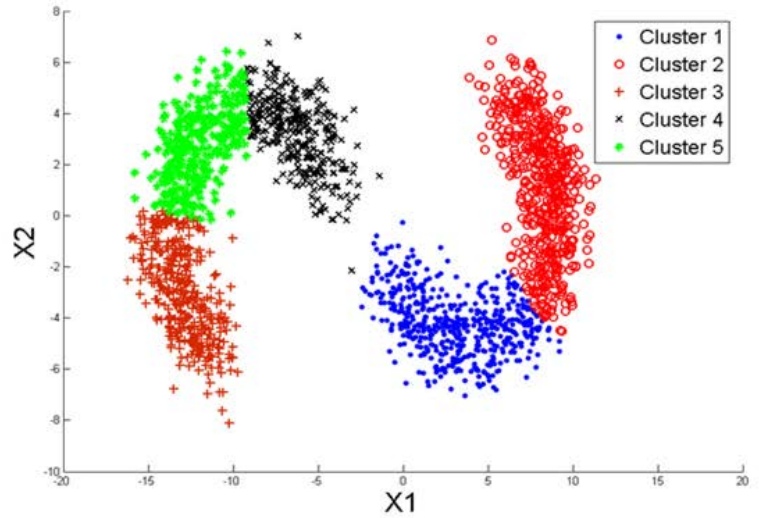

(b)

Figure 2. (a) The two banana-shaped in-control observations and (b) five in-control groups that result after partitioning in-control observations by a $k$-means clustering algorithm $(k=5)$.

Figure 3 summarizes the clustering-based control chart introduced by Kang and Kim (2013).

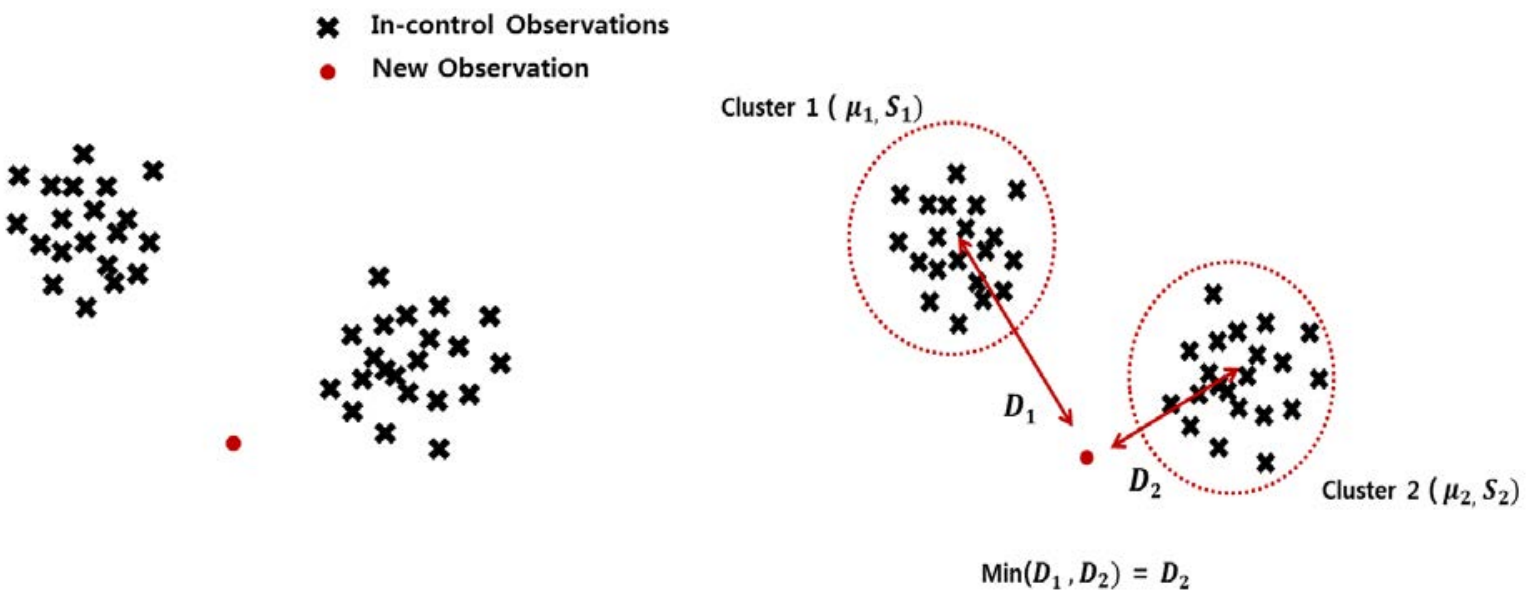

(a)

(b) 
Figure 3. An overall process to calculate the monitoring statistics for the clustering-based chart, based on a clustering algorithm. (a) in-control observations and a new observation and (b) calculation of distances from grouped in-control observations to the new observation.

The distance of an incoming unclassified observation to the center of the $k^{\text {th }}$ cluster, which is a monitoring statistic of the clustering-based control chart, can be calculated as follows:

$$
\tau(x)=\min _{k}\left(x-\mu_{k}\right)^{T} \Sigma_{k}^{-1}\left(x-\mu_{k}\right),
$$

where $x$ is the incoming unclassified observation. $\mu_{k}$ and $\Sigma_{k}$ are, respectively, the mean vector and the covariance matrix from the $k^{\text {th }}$ cluster. Consequently, $\tau(x)$ indicates the minimum abnormality between a given observation and those observations deemed normal. Kang and Kim (2013) used a $k$-means clustering algorithm to identify the natural groupings of in-control observations although any other clustering algorithms can be used. In the $k$-means clustering algorithm, the number of clusters, which is designated as $k$, should be prespecified by the user. In general, one can try various values of $k$ and select the one that yields the best performance for the control chart. Kang and Kim (2013) revealed the different values of $k$ do not significantly affect the result. In the current paper, we used $k=5$, which gave on average the lowest rates of Type I errors in the simulations and real case studies. As for the control limits of the clustering-based control chart, the probability distribution of the monitoring statistic obtained from Equation 4 is unknown. Thus, Kang and Kim (2013) proposed using the bootstrap method to establish the control limit.

In this paper, the time-adaptive clustering-based control chart (TAC-based control chart) is proposed to monitor time-varying and multimodal processes at the same time. When the process 
condition changes gradually, the parameters of the control chart should be updated over time. Figure 4 shows a situation in which the in-control observations change over time. Note that the TAC-based control chart adapts to the change in the most recent observations, which are plotted at the edge of in-control region by updating the mean vectors and covariance structures of each cluster.
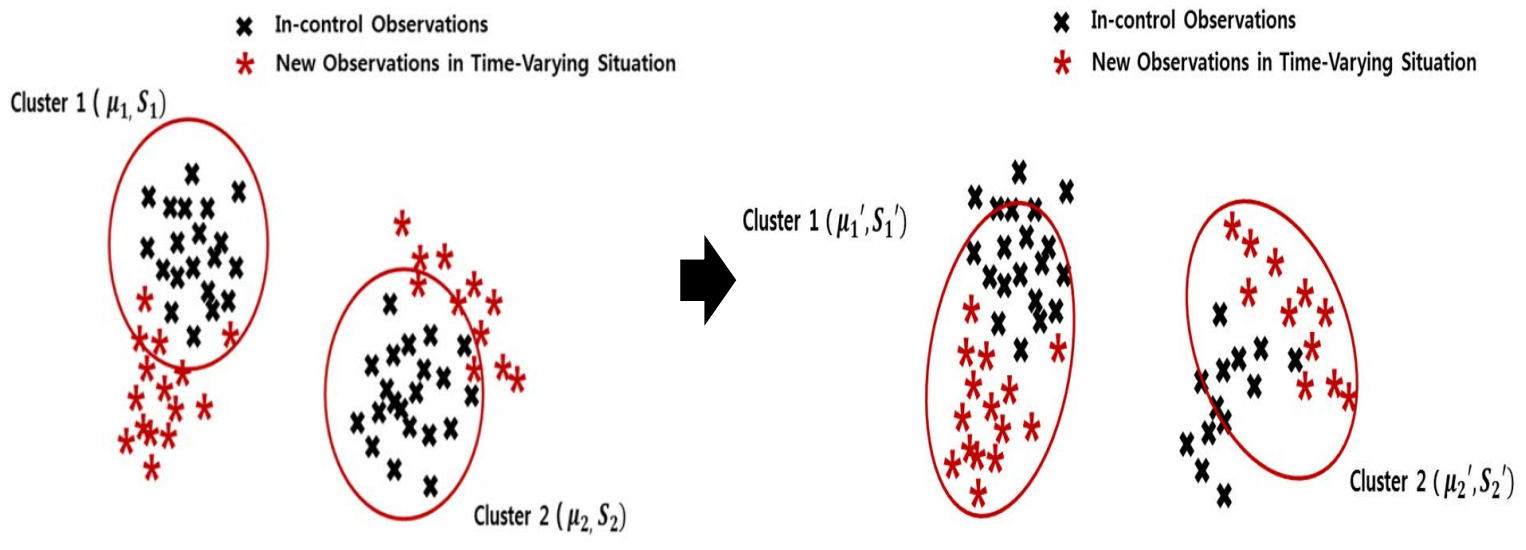

Figure 4. The graphical representation of updating in-control areas by a TAC-based chart.

The updated monitoring statistic of the one-step ahead observation $\left(x^{i+1}\right)$ in a TAC-based control chart can be computed as follows:

$$
\tau\left(x^{i+1}\right)=\min _{k}\left(x^{i+1}-\mu_{k}^{i+1}\right)^{T}\left(\sum_{k}^{i+1}\right)^{-1}\left(x^{i+1}-\mu_{k}^{i+1}\right),
$$

where

$$
\begin{aligned}
& \mu_{k}^{i+1}=\mu_{k}^{i}+\Delta \mu_{k}^{i}, \quad \Delta \mu_{k}^{i}=\delta\left(x^{i+1}-\mu_{k}^{i}\right) I_{k}\left(x^{i+1}\right) \\
& \Sigma_{k}^{i+1}=\Sigma_{k}^{i}+\Delta \Sigma_{k}^{i}, \Delta \Sigma_{k}^{i}=\varphi\left\{\left(x^{i+1}-\mu_{k}^{i}\right)\left(x^{i+1}-\mu_{k}^{i}\right)^{T}-\Sigma_{k}^{i}\right\} I_{k}\left(x^{i+1}\right) .
\end{aligned}
$$

Here, $I_{k}$ is an indicator function defined as follows:

$$
I_{k}\left(x^{i+1}\right)=\left\{\begin{array}{cc}
1, & \text { if } x^{i+1} \in C_{k} \text { and } \tau\left(x^{i+1}\right)<C L_{B} \\
0, & \text { otherwise }
\end{array}\right.
$$


where $C L_{B}$ is the bootstrap-based control limit. An indicator function $I_{k}$ takes the value one when the $i^{\text {th }}$ observation $\left(x^{i}\right)$ belongs to in-control region of the $k^{\text {th }}$ cluster $\left(C_{k}\right)$ and zero otherwise. In summary, the mean vector and covariance structure of each cluster is updated only when a new observation belongs to one of the in-control cluster regions.

From Equation 5, the mean vector and covariance matrix are updated with exponential smoothing scheme to compensate for the limitation of the moving window method that gives the same weights for all observations. In other words, the weights of the proposed method decrease geometrically with the age of the observation. $\Delta \mu_{k}^{i}$ is the amount of mean vector updated, and $\delta$ is a weighting factor $(0 \leq \delta \leq 1)$ of the difference between the current observation and the existing mean vector (Dayal and MacGregor, 1997). Besides, $\Delta \Sigma_{k}^{i}$ is the amount of the updated covariance matrix, and $\varphi$ is a weighting factor $(0 \leq \varphi \leq 1)$ of the difference between the new observation and the existing covariance structure. The larger values of $\delta$ and $\varphi$, the more newly observations should influence the updated monitoring statistics. In general, the choice of weighting parameters is selected empirically. A detailed description of the selection of the parameters is described in Section 4.2.

\section{Simulation study}

\subsection{Simulation setup}

A simulation study was conducted to examine the properties of the proposed TAC-based control chart and to compare it with the existing control charts under various scenarios. The four simulation scenarios are as follows: 
- S1: 5,000 observations from the bivariate multimodal normal distribution (containing five normal distributions) with homogeneous variance structures;

- S2: 2,000 observations from the bivariate multimodal banana-shaped distribution (containing two banana-shaped distributions);

- S3: 1,500 observations from the bivariate multimodal normal distribution (containing three normal distributions) with distinct densities;

- S4: 4,000 observations from the bivariate multimodal normal distribution (containing four normal distributions) with heterogeneous covariance structures over time.

We also generated observations with a time-varying property from each scenario. To monitor and update the monitoring statistic, we also generated the same number of in-control observations, which were gradually shifted by $\theta$. In this study, we set the size of shift $(\theta)$ as 0.01 for S1, S2, and S3, which means that the in-control region can be shifted by 0.01 for new incontrol observation.

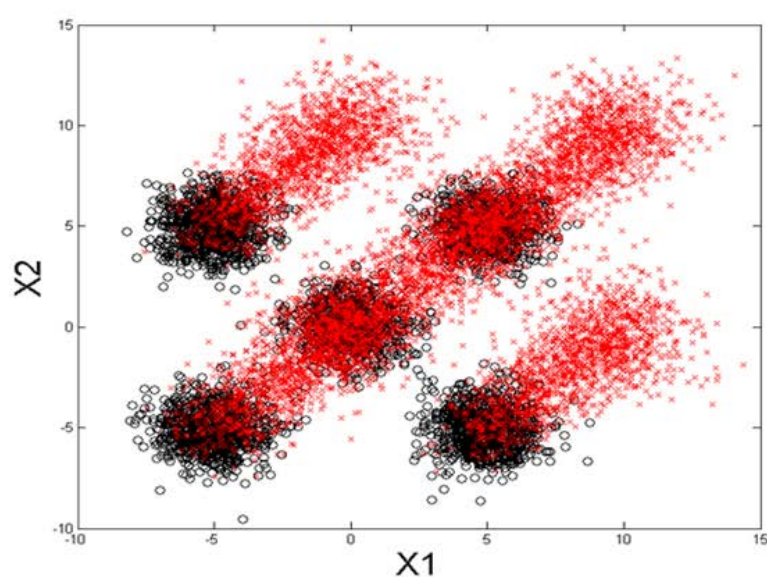

(a)

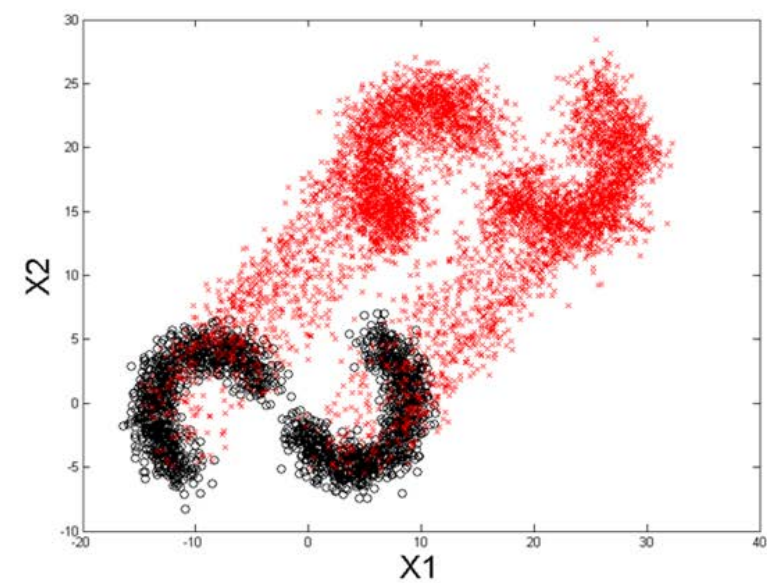

(b) 


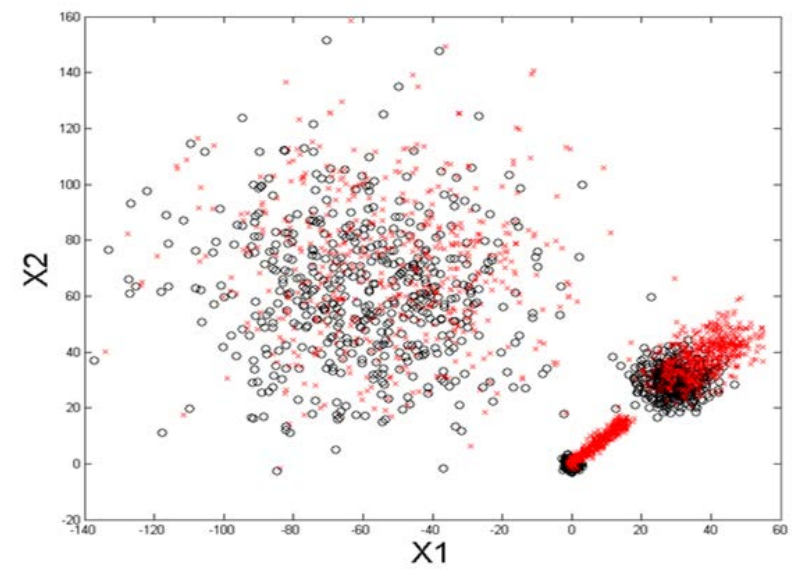

(c)

Figure 5. Simulated in-control observations from (a) S1: the multimodal normal distributions $(\theta=0.01),(b) \mathrm{S} 2$ : the multimodal banana-shaped distribution $(\theta=0.01)$, and (c) S3: the multimodal normal distribution with different densities $(\theta=0.01)$.

Figure 5 (a) shows the observations generated from the multimodal normal distributions containing five individual normal distributions with different means but the same variance structures. Figure 5 (b) shows the multimodal banana-shaped distribution, and Figure 5 (c) shows that the in-control observations are from multimodal normal distributions (three distributions) with very different densities. Note that the banana-shaped distribution represents a nonlinearly distributed dataset with a local pattern.

Furthermore, we generated the in-control observations from multimodal normal distributions whose covariance structures are diversifying over time. Figure 6 demonstrates a graphical representation of covariance transition in S4. In this scenario, we only set the different covariance matrices of each mode as three steps and the mean vectors of multimodal distributions as constant as a way to examine the effect of covariance update. For each transition step, we generated 1,000 observations that have different covariance matrices; this means that 
the variance of each in-control group becomes larger as time passes. For example, the covariance matrix of the first cluster in S4 (the left group in Figure 6) is $\left[\begin{array}{ll}1 & 0 \\ 0 & 1\end{array}\right]$ at the initial step, however, it is being transformed into $\left[\begin{array}{ll}2 & 0 \\ 0 & 2\end{array}\right]$ with larger variability at the final step.
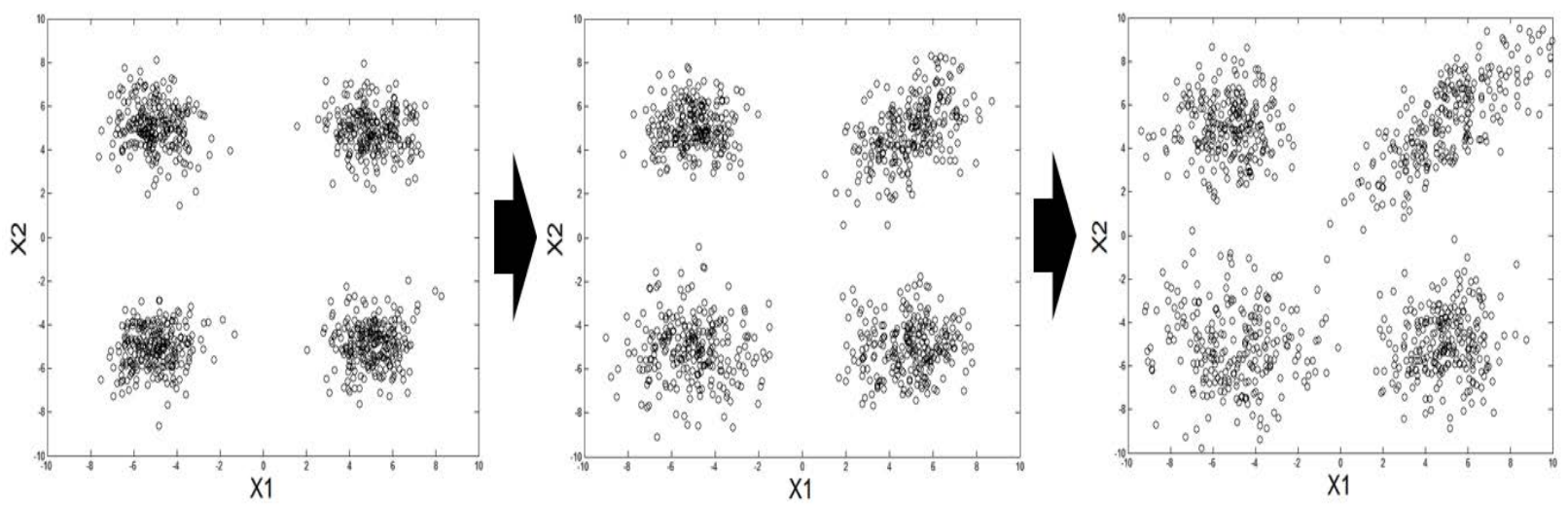

Figure 6. Three transition steps of simulated in-control observations from S4, including the multimodal normal distributions with heterogeneous covariance structures that are changing over time.

\subsection{Parameter setting}

As stated in Section 3, the parameters $\delta$ and $\varphi$ affect the performance of the proposed TACbased control chart. Tables 1, 2, 3, and 4 represent the actual Type I error rates of the TAC-based chart under S1, S2, S3, and S4. We compared the performances by changing the weighting parameters $(\delta$ and $\varphi)$, given 0.05 as the expected Type I error rate. We selected the best parameters that yielded an actual false alarm rate closest to the expected false alarm rate. For example, in the S1 scenario, the parameters $\delta=0.7$ and $\varphi=0.01$ were considered the best because a TAC-based control chart with these parameters produced values that were the most similar to the expected and actual false alarm rates (Table 1). As for the parameter $\varphi$, we adjusted it over a range between 0.01 and 0.09 because the false alarm rates increased abruptly if $\varphi$ exceeded 0.1 . In contrast with the updating mean vector $(\delta)$, a large update on the covariance 
matrix (i.e., large $\varphi$ ) makes the control chart more unreliable because the covariance structure is generally shifted through longer period compared to mean shift.

Table 1. Actual Type I error rates with changing the weighting parameters of a TAC-based chart under the simulated mean shift case (S1) when the expected Type I error rate is 0.05 . The boldface indicates the optimal set of parameters.

\begin{tabular}{cccccccccc}
\hline \multicolumn{1}{c}{$\delta$} & 0.1 & 0.2 & 0.3 & 0.4 & 0.5 & 0.6 & $\mathbf{0 . 7}$ & 0.8 & 0.9 \\
\hline $\mathbf{0 . 0 1}$ & 0.058 & 0.057 & 0.055 & 0.056 & 0.056 & 0.055 & $\mathbf{0 . 0 5 3}$ & 0.055 & 0.060 \\
0.02 & 0.059 & 0.060 & 0.059 & 0.059 & 0.059 & 0.058 & 0.056 & 0.057 & 0.059 \\
0.03 & 0.063 & 0.064 & 0.062 & 0.063 & 0.063 & 0.062 & 0.059 & 0.061 & 0.060 \\
0.04 & 0.067 & 0.066 & 0.066 & 0.064 & 0.065 & 0.064 & 0.062 & 0.062 & 0.061 \\
0.05 & 0.070 & 0.070 & 0.069 & 0.068 & 0.067 & 0.066 & 0.066 & 0.065 & 0.063 \\
0.06 & 0.073 & 0.074 & 0.074 & 0.072 & 0.070 & 0.068 & 0.069 & 0.067 & 0.066 \\
0.07 & 0.077 & 0.076 & 0.077 & 0.076 & 0.072 & 0.071 & 0.072 & 0.071 & 0.070 \\
0.08 & 0.079 & 0.079 & 0.081 & 0.079 & 0.075 & 0.075 & 0.074 & 0.075 & 0.074 \\
0.09 & 0.082 & 0.084 & 0.086 & 0.083 & 0.079 & 0.078 & 0.078 & 0.080 & 0.078 \\
\hline
\end{tabular}

Table 2. Actual Type I error rates with changing of the weighting parameters of a TAC-based chart under the simulated mean shift case (S2) when the expected Type I error rate is 0.05 . The boldface indicates the optimal set of parameters.

\begin{tabular}{|llllllllll}
\hline$\delta$ & 0.1 & 0.2 & $\mathbf{0 . 3}$ & 0.4 & 0.5 & 0.6 & 0.7 & 0.8 & 0.9 \\
\hline
\end{tabular}




\begin{tabular}{lllllllllll}
\hline 0.01 & 0.049 & 0.045 & 0.045 & 0.041 & 0.043 & 0.044 & 0.044 & 0.044 & 0.044 \\
0.02 & 0.048 & 0.049 & 0.048 & 0.049 & 0.050 & 0.051 & 0.051 & 0.052 & 0.051 \\
0.03 & 0.048 & 0.049 & 0.049 & 0.048 & 0.049 & 0.049 & 0.055 & 0.056 & 0.057 \\
$\mathbf{0 . 0 4}$ & 0.053 & 0.054 & $\mathbf{0 . 0 5 0}$ & 0.049 & 0.053 & 0.052 & 0.052 & 0.053 & 0.053 \\
0.05 & 0.049 & 0.051 & 0.048 & 0.053 & 0.053 & 0.054 & 0.053 & 0.052 & 0.051 \\
0.06 & 0.049 & 0.048 & 0.047 & 0.049 & 0.048 & 0.054 & 0.048 & 0.052 & 0.054 \\
0.07 & 0.056 & 0.058 & 0.053 & 0.053 & 0.053 & 0.054 & 0.054 & 0.055 & 0.052 \\
0.08 & 0.049 & 0.050 & 0.048 & 0.047 & 0.050 & 0.050 & 0.049 & 0.050 & 0.049 \\
& & & & & & & & & & \\
0.09 & 0.056 & 0.056 & 0.055 & 0.057 & 0.057 & 0.057 & 0.058 & 0.060 & 0.063 \\
\hline
\end{tabular}

Table 3. Actual Type I error rates with changing of the weighting parameters of a TAC-based chart under the simulated mean shift case (S3) when the expected Type I error rate is 0.05 . The boldface indicates the optimal set of parameters.

\begin{tabular}{|c|c|c|c|c|c|c|c|c|c|}
\hline 0.01 & 0.050 & 0.039 & 0.033 & 0.034 & 0.029 & 0.030 & 0.031 & 0.032 & 0.047 \\
\hline 0.02 & 0.043 & 0.042 & 0.034 & 0.033 & 0.030 & 0.031 & 0.029 & 0.030 & 0.033 \\
\hline 0.03 & 0.045 & 0.044 & 0.039 & 0.034 & 0.035 & 0.032 & 0.032 & 0.030 & 0.032 \\
\hline 0.04 & 0.048 & 0.043 & 0.039 & 0.038 & 0.038 & 0.033 & 0.033 & 0.033 & 0.034 \\
\hline 0.05 & 0.053 & 0.060 & 0.056 & 0.053 & 0.051 & 0.049 & 0.045 & 0.046 & 0.044 \\
\hline 0.06 & 0.074 & 0.062 & 0.055 & 0.057 & 0.056 & 0.051 & 0.050 & 0.047 & 0.046 \\
\hline 0.07 & 0.074 & 0.070 & 0.059 & 0.067 & 0.059 & 0.054 & 0.053 & 0.050 & 0.054 \\
\hline
\end{tabular}




\begin{tabular}{llllllllll}
0.08 & 0.080 & 0.071 & 0.064 & 0.069 & 0.065 & 0.065 & 0.059 & 0.055 & 0.067 \\
0.09 & 0.080 & 0.076 & 0.071 & 0.083 & 0.076 & 0.069 & 0.067 & 0.063 & 0.074 \\
\hline
\end{tabular}

Table 4. Actual Type I error rates with changing of the weighting parameters of a TAC-based chart under the simulated covariance shift case (S4) when the expected Type I error rate is 0.05 . The boldface indicates the optimal set of parameters.

\begin{tabular}{|c|c|c|c|c|c|c|c|c|c|}
\hline & 0.1 & 0.2 & 0.3 & 0.4 & 0.5 & 0.6 & 0.7 & 0.8 & 0.9 \\
\hline 0.01 & 0.064 & 0.066 & 0.065 & 0.065 & 0.066 & 0.069 & 0.070 & 0.071 & 0.070 \\
\hline 0.02 & 0.059 & 0.059 & 0.059 & 0.061 & 0.059 & 0.059 & 0.061 & 0.061 & 0.061 \\
\hline 0.03 & 0.059 & 0.058 & 0.059 & 0.060 & 0.061 & 0.057 & 0.060 & 0.061 & 0.057 \\
\hline 0.04 & 0.059 & 0.059 & 0.059 & 0.062 & 0.063 & 0.062 & 0.063 & 0.063 & 0.060 \\
\hline 0.05 & 0.063 & 0.062 & 0.062 & 0.063 & 0.066 & 0.069 & 0.063 & 0.063 & 0.061 \\
\hline 0.06 & 0.065 & 0.064 & 0.064 & 0.066 & 0.067 & 0.072 & 0.068 & 0.065 & 0.069 \\
\hline 0.07 & 0.066 & 0.065 & 0.066 & 0.066 & 0.069 & 0.075 & 0.069 & 0.068 & 0.071 \\
\hline 0.08 & 0.070 & 0.069 & 0.068 & 0.070 & 0.070 & 0.076 & 0.074 & 0.070 & 0.075 \\
\hline 0.09 & 0.073 & 0.072 & 0.071 & 0.070 & 0.071 & 0.075 & 0.074 & 0.072 & 0.078 \\
\hline
\end{tabular}

\subsection{Simulation results}

4.3.1 Comparison results between a clustering-based chart and a time-adaptive clustering-based chart 


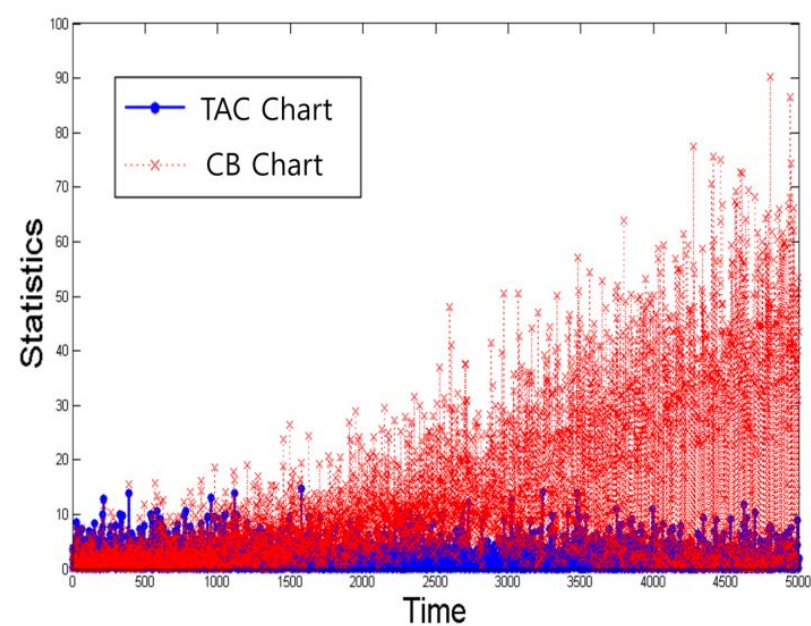

(a)

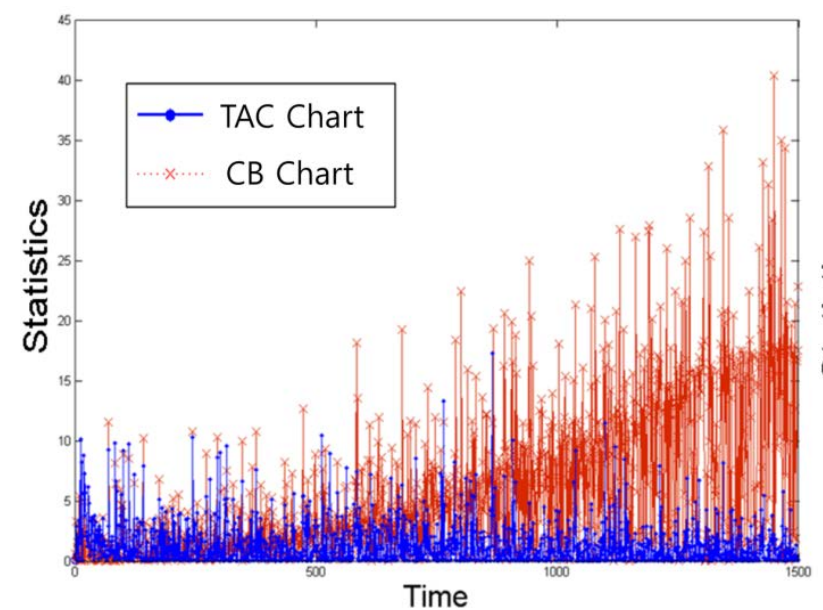

(c)

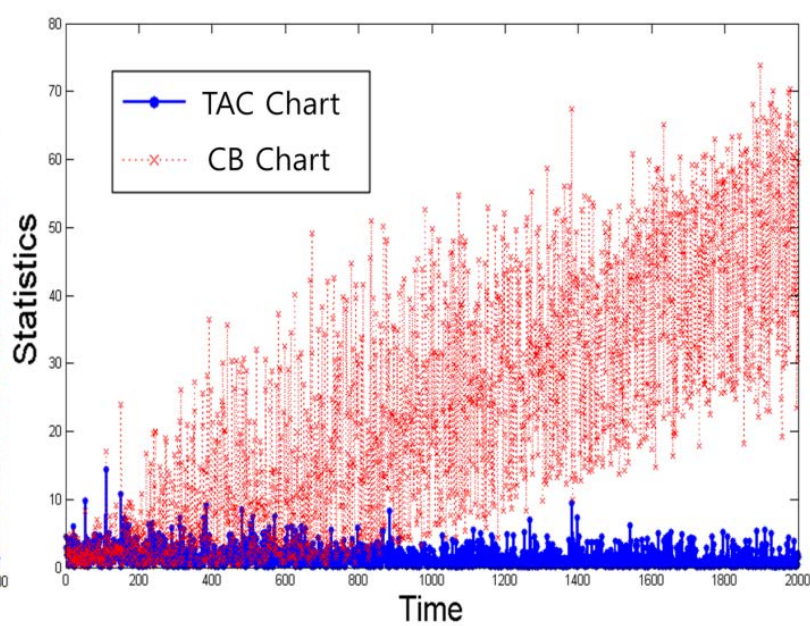

(b)

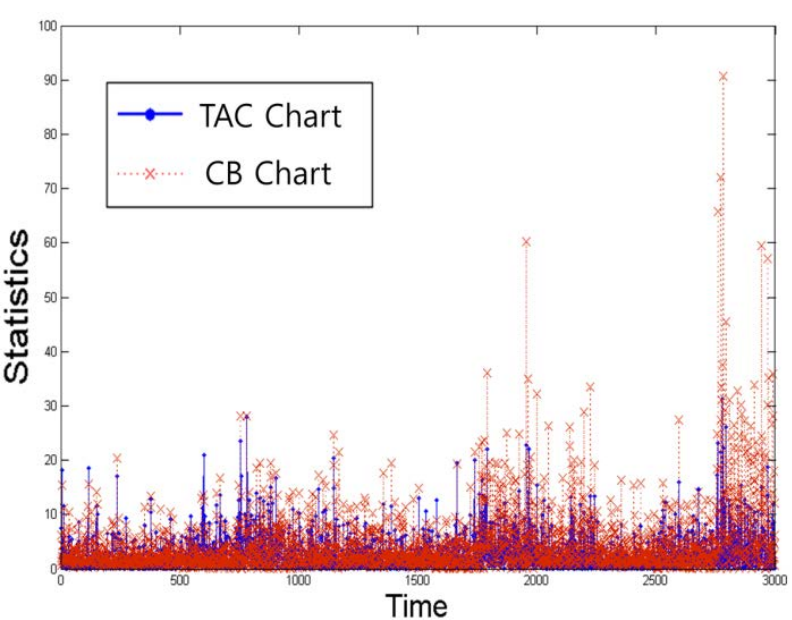

(d)

Figure 7. Comparisons of monitoring statistics between the clustering-based chart and the TACbased chart in four simulation scenarios: (a) S1, (b) S2, (c) S3, and (d) S4.

Figure 7 displays the monitoring statistics of the clustering-based chart and TAC-based chart over time. It can be observed that the clustering-based chart could not appropriately accommodate time-varying patterns such as changes in the mean vector or covariance matrix. Consequently, numerous false alarms were generated. On the other hand, the monitoring 
statistics of the proposed TAC-based control chart are relatively stable with an increase of shift size, leading to fewer false alarms than with the clustering-based chart. These results clearly show the advantage of the proposed chart.

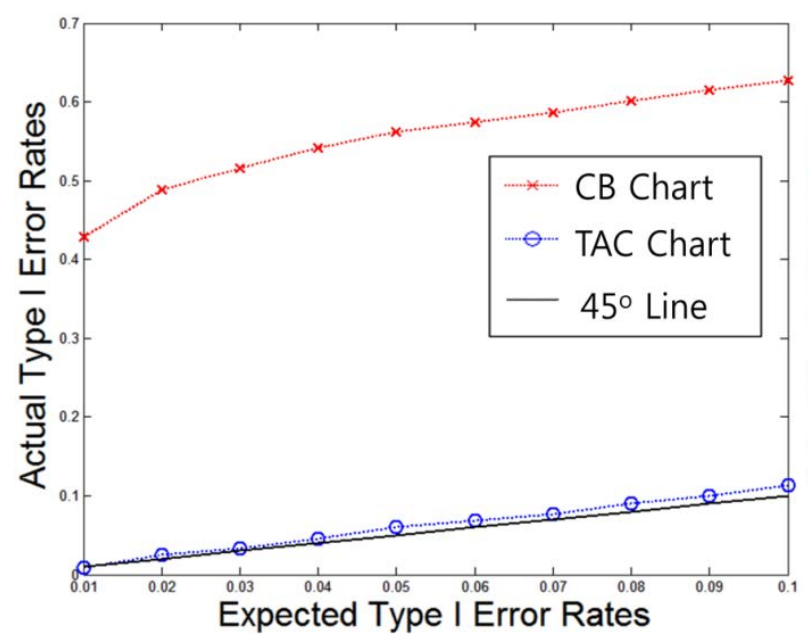

(a)

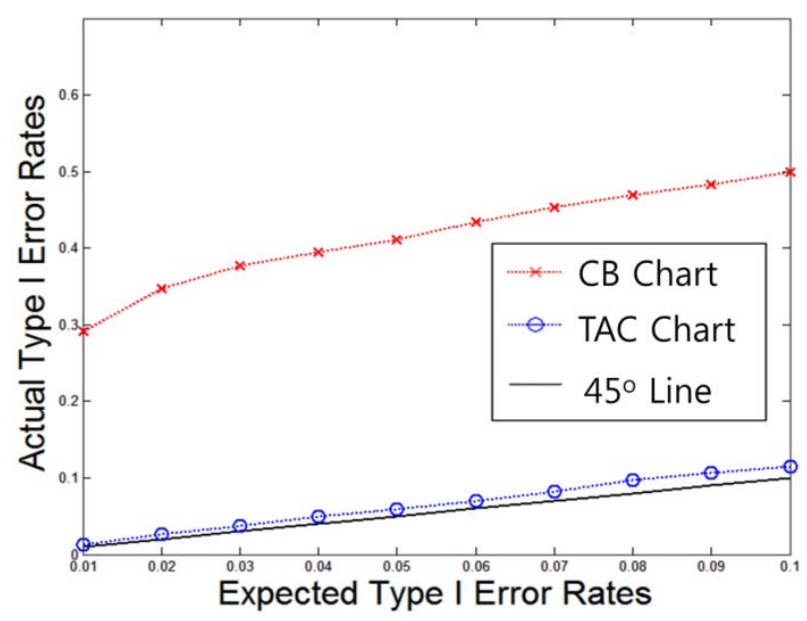

(c)

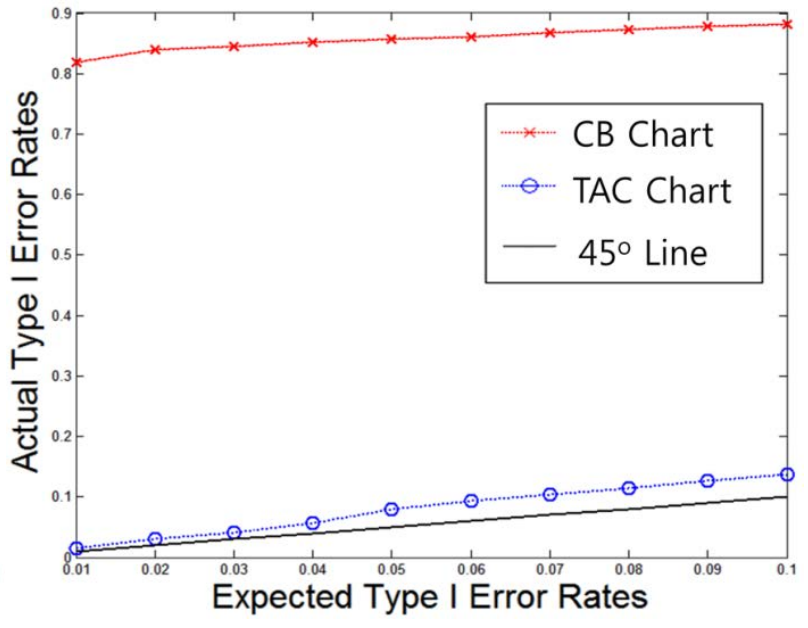

(b)

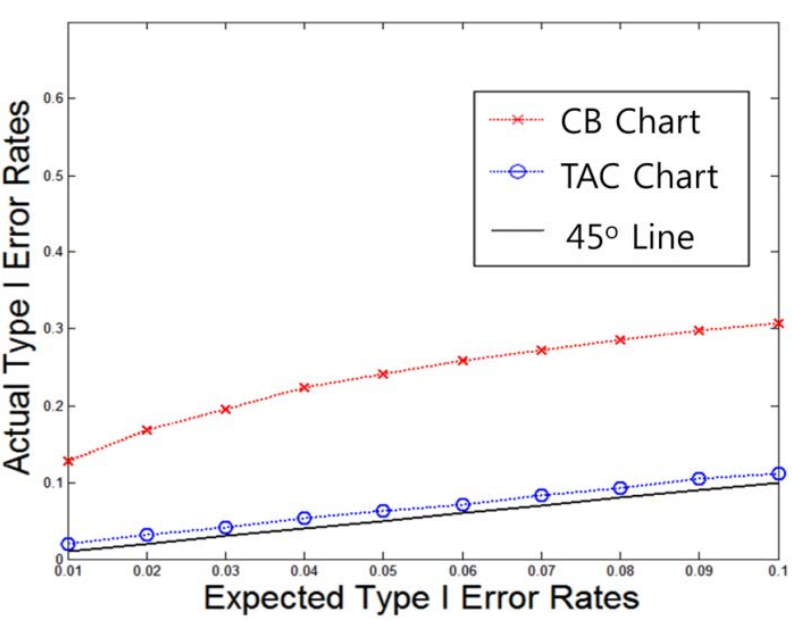

(d)

Figure 8. Changes in average values of actual false alarm rates corresponding to the different expected false alarm rates of two control charts with different simulation cases: (a) S1, (b) S2, (c)

$$
\text { S3, and (d) S4. }
$$


Figure 8 shows the performance of two control charts with different expected Type I error rates $(0.01 \sim 0.1)$. It is preferable that the expected Type I error rate of a control chart is the same as or as close as possible to the actual Type I error rate. In other words, control charts whose two dimensional points of the expected and actual Type I error rates are plotted near the 45-degree line should be preferred. Figure 8 shows that the points from the TAC-based chart lie near the 45-degree line, but the points from the clustering-based chart deviate significantly from the 45degree line. This result indicates that proposed chart can properly control the false alarm rate in time-varying situations, and it thus significantly reduces the number of false alarms. However, we thought that the comparative results between the clustering-based and TAC charts are not surprising because the proposed TAC chart has an updating feature, while the clustering-based chart does not. Our main intent of this comparison is to demonstrate whether or not the updating feature of the proposed chart works properly. In the next section, we compare the proposed TAC chart with time-adaptive GMM-based chart, the lasted technique for handling time-varying and multimodal situations.

In evaluating the performance of control charts, average run length (ARL) is an widely used measure. Although ARL is a practical measure in evaluating the performance of the processes, we thought that in our case the use of ARL was not appropriate because of its correlated and time-dependent process observations that could make its results unreliable (Montgomery, 2005). Therefore, we only compared the performance of control charts in terms of false alarm rates (Type I error rates).

4.3.2 Comparison results between time-adaptive GMM chart and time-adaptive clustering-based chart 
The performance of the TAC-based chart is compared with that of an existing time-adaptive GMM-based chart that was introduced to cope with time-varying and multimodal process conditions. In this study, we compared our proposed TAC-based chart with Xie and Shi (2012)'s latest GMM monitoring method introduced in Section 2.

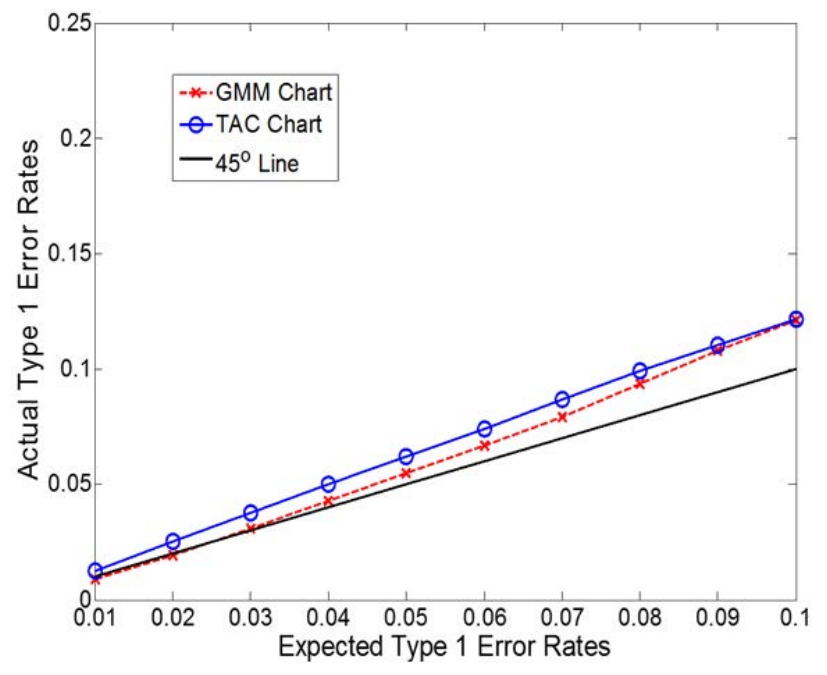

(a)

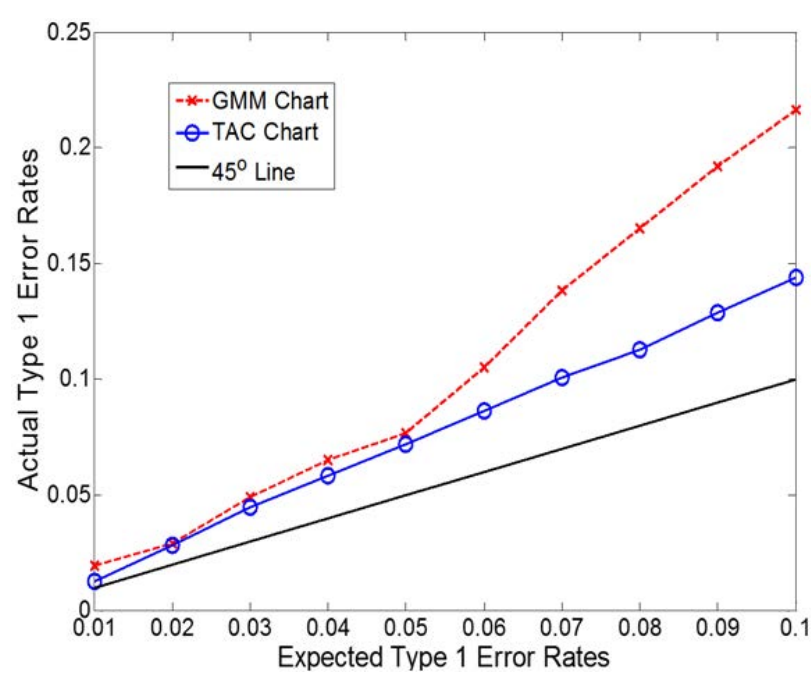

(c)

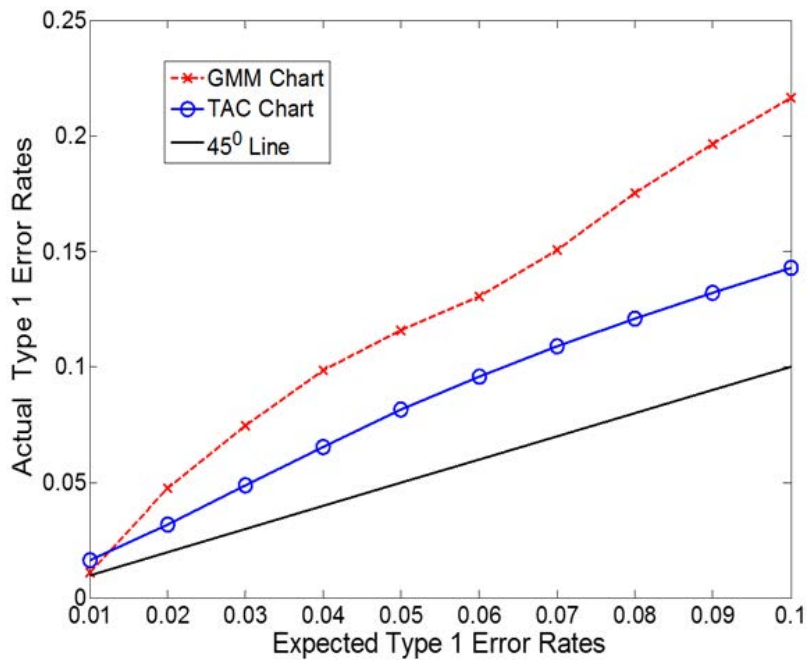

(b)

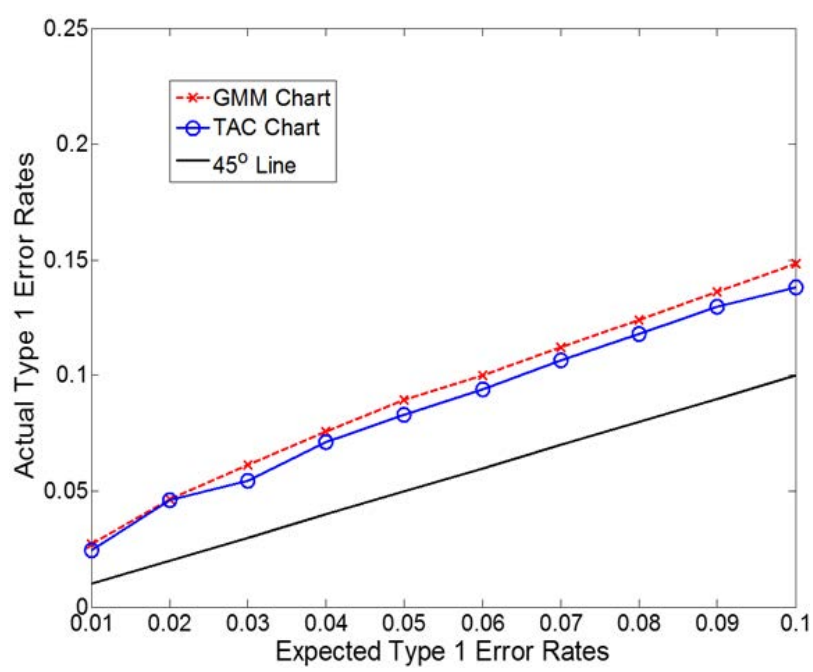

(d) 
Figure 9. Changes in average values of actual false alarm rates corresponding to the different expected false alarm rates of a TAC-based chart and a GMM chart with different simulation

$$
\text { cases: (a) S1, (b) S2, (c) S3, and (d) S4. }
$$

Figure 9 shows the performance of two control charts with different expected Type I error rates $(0.01 \sim 0.1)$. Note that the points from the TAC-based chart lie closer to the 45 -degree line and are stably plotted, while the points from the GMM-based chart deviate from the 45-degree line. Especially, in S2 and S3, the false alarm rates of the GMM-based chart are relatively high because the GMM-based chart has a major limitation in its frequent insufficient robustness to deal with outliers in data (Svensen and Bishop, 2005). In S2 (Figure 9 (b)), the multimodal banana-shaped distribution not only has a nonlinear pattern, but also its observations are nonnormally distributed; as a result, a time-adaptive GMM-based chart may establish an incorrect parametric control limit that is incapable of controlling reasonable false alarm rates. The control limit of a GMM-based chart is based on an F-distribution, which frequently leads to poor performance when in-control observations do not follow a multivariate normal distribution (Phaladiganon, 2012). Compared with GMM-based chart, the main advantage of a TAC-based chart is not only the correctness of its monitoring statistic, but also its rational false alarm rates that are the result of its control limit that is calculated by a bootstrapping method. Moreover, as shown in S3 (Figure 9 (c)), false alarm rates of GMM-based chart is constantly larger than the proposed TAC-based chart when the observations have distinct densities among in-control groups. This denotes that in a variety of time-varying and multimodal situations, the proposed TAC-based chart has a more reasonable number of false alarms than the GMM-based chart.

It should be noted that although the performance of the proposed method is generally better than that of the other charts, the actual Type I error rates were still larger than the expected Type 
I error rates. In fact, it is very challenging to control the actual Type I error rates to be the exactly same as the expected Type I error rates in time-varying situations. We have to admit that this is a limitation in our current algorithm. One idea that we could try is as follows: Currently, we update the parameters for every new observation that may not be able to affect the change of the parameters properly. Thus, we would use enough observations (e.g., batch) to update the parameters rather than using every single observation. Of course, in this case, we should determine the size of observations (i.e., batch size).

Figure 10 indicates the monitoring statistics of the TAC-based chart and GMM-based chart in S3. Notice that GMM-based chart could not appropriately reflect distinct densities among incontrol groups. On the other hand, monitoring statistics of TAC-based chart are stable to the distinct density or time-varying conditions. This denotes that the amount of false alarms with proposed TAC-based chart is more reasonable than GMM-based chart in the time-varying and multimodal situations.

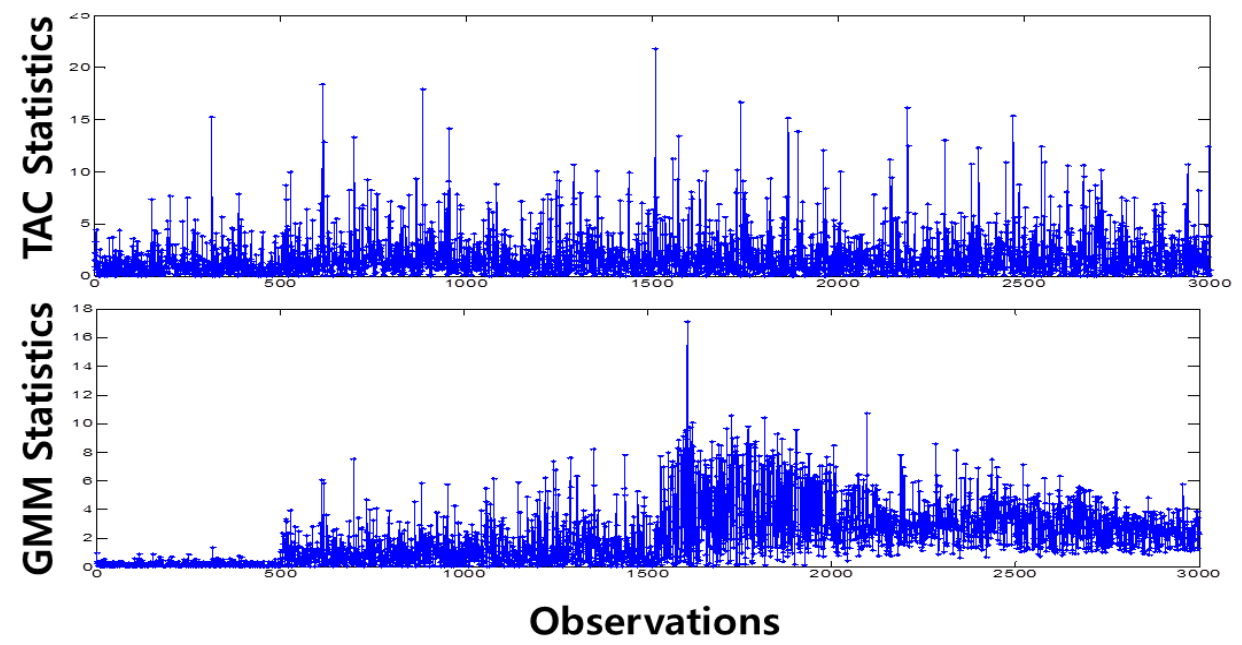

Figure 10. Comparisons of monitoring statistics between the TAC-based chart and the GMM-based chart in S3. 


\section{TFT-LCD case study}

In this section, we applied the proposed TAC-based control chart to real case from the TFT-LCD process. As shown in Figure 1 of Introduction, the observations from the process were grouped based on different manufacturing tools, indicating a multimodal process.

\subsection{Description of TFT-LCD manufacturing process}

The manufacturing of a TFT-LCD consists of a sequence of processes: deposition, cleaning, photo resistance coating, exposure, developing, etching, strip, and inspection. Each process requires several repetitions, leading to multilayered products. Figure 11 shows an overview of the TFT-LCD manufacturing process.

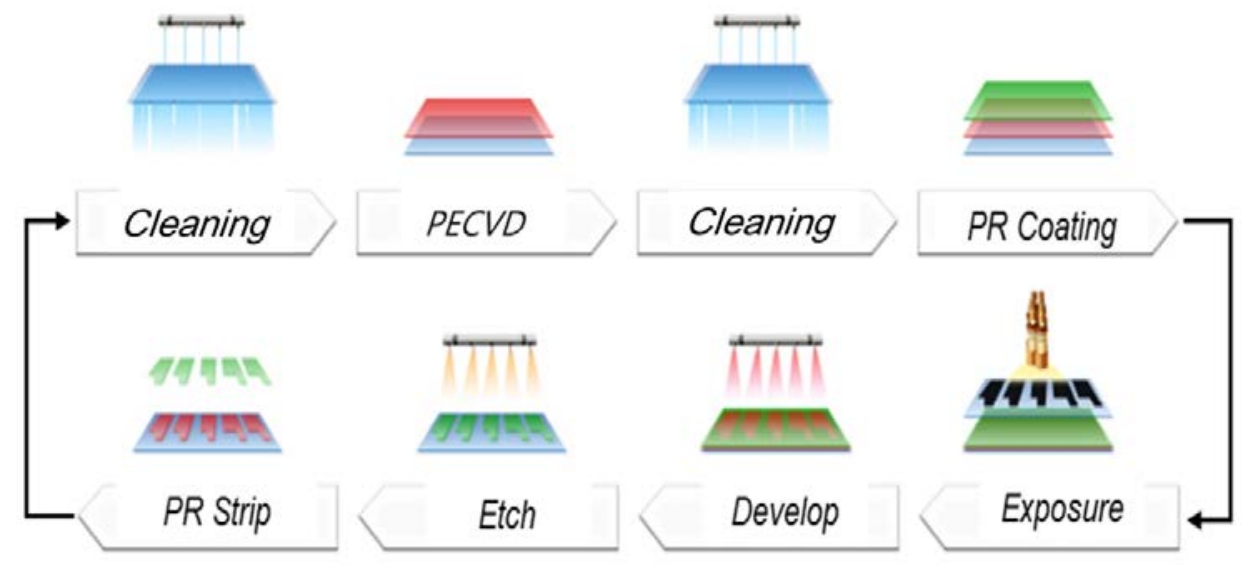

Figure 11. An overview of TFT-LCD processes.

The plasma enhanced chemical vapor deposition (PECVD) process, one of the deposition processes, creates silicon nitride (Sinx), amorphous silicon (a-Si), and $\mathrm{n}+$ silicon films that are used for semiconductor and insulator films. This process is recognized as critical because this step determines the performance of a transistor component. In this study, we focused on the Sinx deposition step in the PECVD process. This step creates a layer of gate insulator designed to 
prevent short circuits. The Sinx deposition step involves a complex procedure performed with a process chamber. In the Sinx step, thin films are deposited on a substrate in a process in which the film material transforms from a gas into a solid state. Some chemical reactions are involved in this process after plasma is created from the reacting gases. The plasma is created by an Radio Frequency (RF) power discharge between two electrodes where the space between them is filled with reacting gases. Moreover, plasma processing is controlled within a range of pressures between a few millitorr and a few Torr. The quality of a Sinx film can be determined by a number of quality characteristics, such as gas flow rates, chamber pressure, RF power, and temperature. These quality characteristics are responsible for equipment failure as well as for the quality of the final products. Therefore, an efficient and reliable monitoring tool is required to detect variations caused by various causes in the processes so that corrective action can be taken before quality deteriorates (Kang et al., 2012).

\subsection{Experimental result of the case study}

For the experiment, we used 1,000 observations, each characterized by 42 quality characteristics from the in-control PECVD process. To evaluate the performance of the proposed TAC-based control chart, we divided the in-control observations into two parts. The first in-control observations (from $1^{\text {st }}$ to the $600^{\text {th }}$ observation) were used to estimate the initial parameters (mean and covariance). The second group of in-control observations (from the $601^{\text {th }}$ to the $1,000^{\text {th }}$ observation) was used to evaluate the control charts in terms of false alarms. 

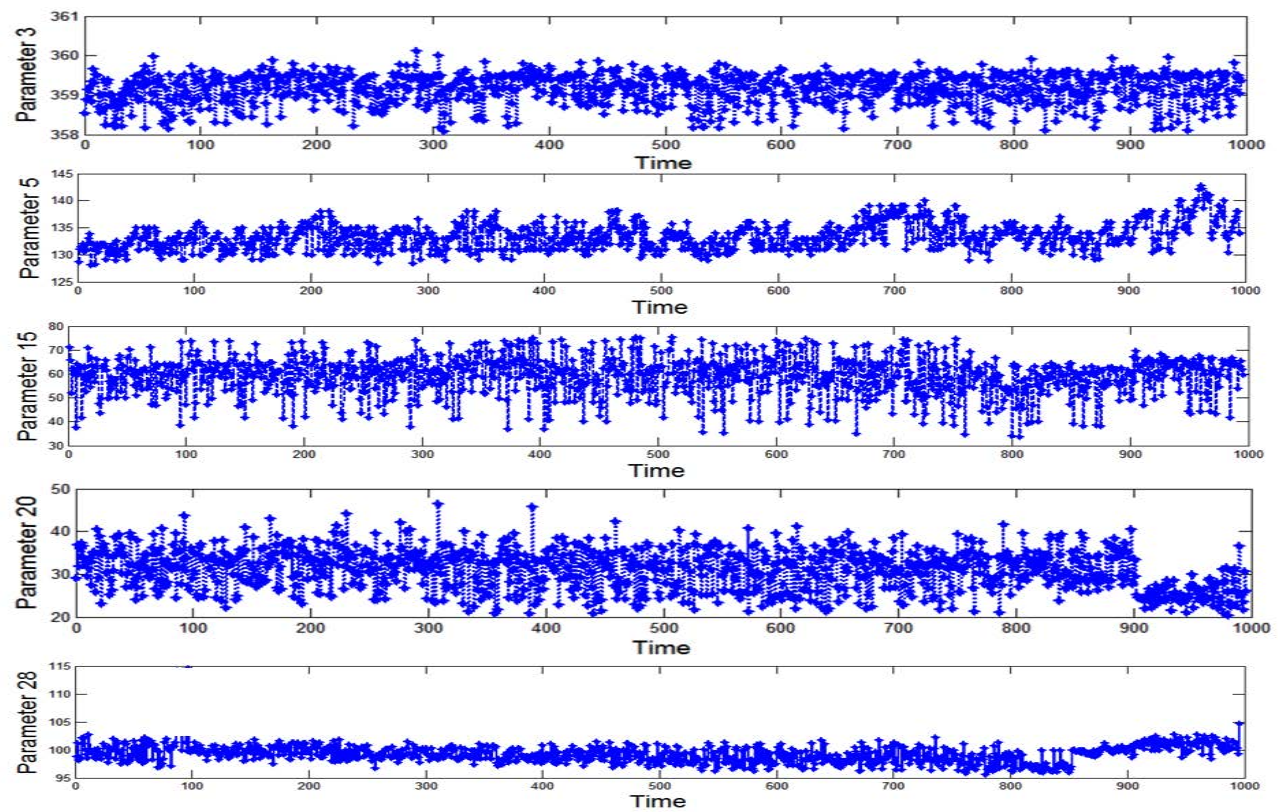

Figure 12. An example of time-varying variables in the TFT-LCD process

Figure 12 shows an example of five quality characteristics that exhibit time-varying patterns. We were informed by the engineers that the process shift starts from the $601^{\text {th }}$ observation. In this process condition, the original clustering-based chart may produce a large number of false alarms because it does not take time-varying patterns into account. Please note that the names of the variables and their explanations are not revealed in this paper because of confidentiality agreement with the company that provided this dataset. 


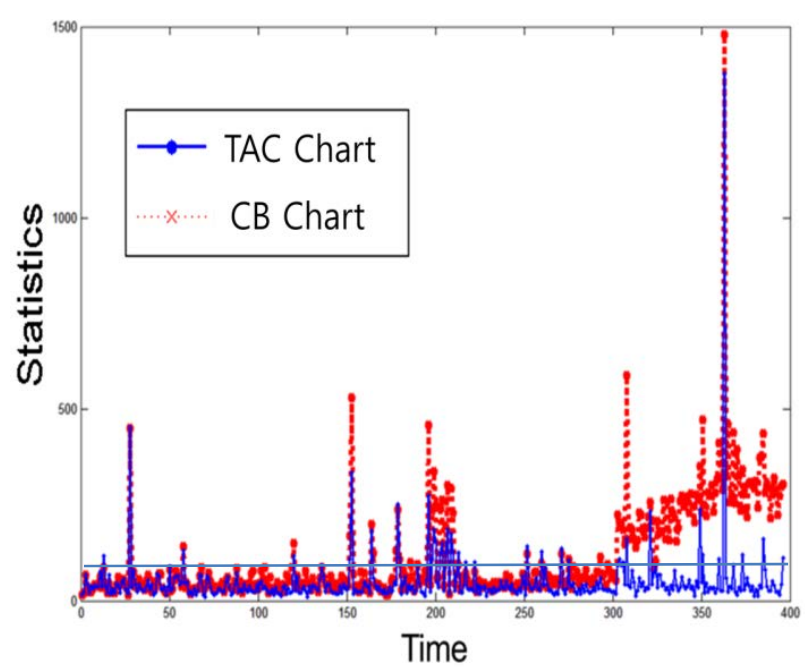

(a)

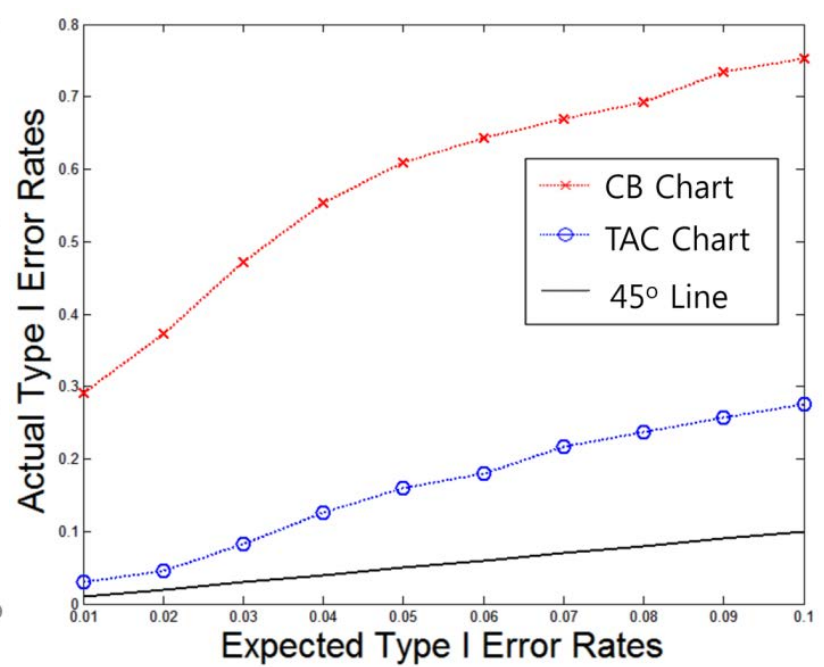

(b)

Figure 13. A comparison between clustering-based and TAC-based control charts with TFTLCD process data (observations from the $601^{\text {th }}$ to the $1,000^{\text {th }}$ ): (a) monitoring statistics from two control charts, (b) actual false alarm rates corresponding to expected false alarm rates.

The monitoring statistics of the second part of the in-control observations from both the original clustering-based chart and the proposed chart are shown in Figure 13 (a). Most of the monitoring statistics of the original clustering-based chart are higher than those of the proposed TAC-based chart, showing a stable pattern over time. This clearly indicates that the original clustering-based chart produced more false alarms than the proposed chart. Figure 13 (b) shows the behavior of expected Type I error rates of the clustering-based and the proposed TAC-based charts over a range of expected Type I error rates from 0.01 to 0.1 . The chart with similar actual and expected false alarm rates would be considered the better one. Figure 13 (b) shows that the proposed TAC-based chart produced more similar values between the actual and expected false alarm rates than the original clustering-based chart. 

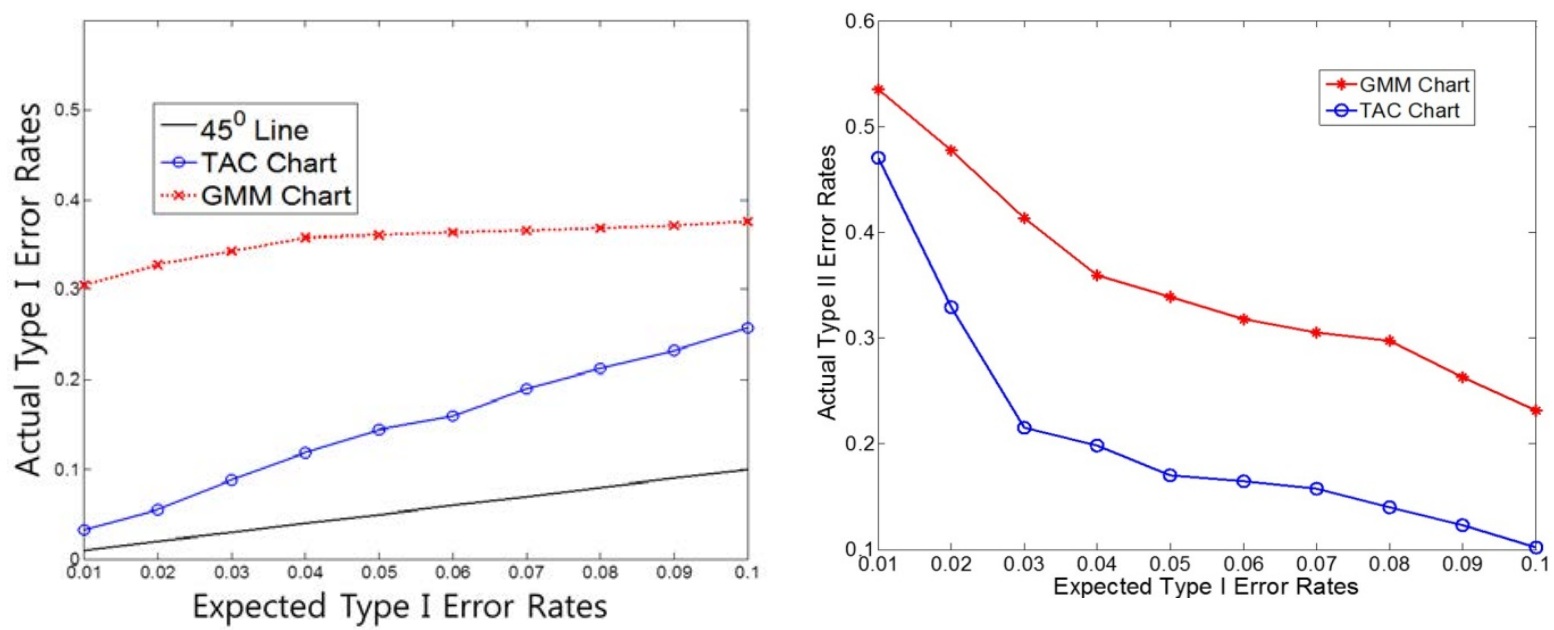

Figure 14. A comparison between TAC-based charts a GMM-based chart with TFT-LCD process data: (a) actual false alarm rates corresponding to expected false alarm rates (observations from the $601^{\text {th }}$ to the $1,000^{\text {th }}$ ), (b) actual Type II error rates corresponding to expected false alarm rates (observations from the $1,001^{\text {th }}$ to the $2,000^{\text {th }}$ ).

Furthermore, as shown in the simulation section (Section 4), the performances of TAC-based and GMM-based charts are evaluated with a TFT-LCD case study. Like the results of simulation studies, Figure 14 (a) shows that the proposed TAC-based chart performs better than the existing GMM-based chart in actual false alarm rates. We also compared the proposed TAC-based control charts with GMM-based charts in terms of Type II error rates with 1,000 out-of-control observations. As mentioned earlier in this section, we were also informed that the faults in the process were occurred from the $1,001^{\text {th }}$ observation to the $2,000^{\text {th }}$ observation. Figure 14 (b) indicates that the proposed TAC-based chart yielded significantly smaller Type II error rates compared to GMM charts. 


\section{Concluding remarks}

In the present study, we have proposed the TAC-based control chart to address the multimodal and time-varying process situations. Because the proposed chart can promptly adjust to timevarying conditions such as a change in a mean vector or a covariance matrix, unwanted false alarms are expected to be reduced in the manufacturing processes. The results from the simulation and case study from the TFT-LCD process showed that the proposed chart outperformed the existing control charts. Although the current study focuses primarily on the issue of reducing the rate of false alarms in the manufacturing process, this idea can be extended to an adaptive strategy for reducing the misdetection rate. Furthermore, we believe that the results of applying other clustering algorithm-based monitoring methods to the time-varying approach pose an interesting issue.

\section{Acknowledgements}

We sincerely thank the editor and two reviewers for their constructive comments and suggestions, which greatly improved the quality of the paper. This research was supported by Brain Korea PLUS and Basic Science Research Program through the National Research Foundation of Korea funded by the Ministry of Science, ICT and Future Planning (2013007724).

\section{References}

1. Bersimis, S., Psarakis, S. and Panaretos, J., 2006. "Multivariate Statistical Process Control Charts: An Overview.” Quality and Reliability Engineering International, 23(5), 517-543. 
2. Chakraborti, S., P., van der Lann, and Bakir, S. T. 2001, "Nonparametric Control Charts: An Overview and Some Results.” Journal of Quality Technology, 33, 304-315.

3. Chongfuangprinya, P., Kim, S. B., Park, S. K., and Sukchotrat, T. 2011, "Integration of support vector machines and control charts for multivariate process monitoring." Journal of Statistical Computation and Simulation, 81(9), 1157-1173.

4. Choi, S. W., Martin, E. B., Morris, A. J., and Lee, I. B. 2006, "Adaptive multivariate statistical process control for monitoring time-varying processes." Industrial and Engineering Chemistry Research, 45(9), 3108-3118.

5. Choi, S. W., Park, J. H., and Lee, I. B., 2004, "Process monitoring using a Gaussian mixture model via principal component analysis and discriminant analysis." Computers \& chemical engineering, 28(8), 1377-1387.

6. Chou, Y. M., Mason,R. L. and Young, J. C. 2001, "The Control Chart for Individual Observations from a Multivariate Non-normal Distribution." Communications in Statistics-Simulation and Computation 30 (8-9), 1937-1949.

7. Dayal, B. S., and MacGregor, J. F. 1997, "Recursive exponentially weighted PLS and its applications to adaptive control and prediction." Journal of Process Control, 7(3), 169179.

8. Figueiredo, M. A., and Jain, A. K., 2002, "Unsupervised learning of finite mixture models.” Pattern Analysis and Machine Intelligence, IEEE Transactions on, 24(3), 381396.

9. Ge, Z., and Song, Z. 2008, “Online monitoring of nonlinear multiple mode processes based on adaptive local model approach.” Control Engineering Practice, 16(12), 14271437. 
10. Hwang, W., Runger, G. and Tuv, E., 2007, "Multivariate statistical process control with artificial contrasts.” IIE Transactions, 39, 659-669.

11. Kang, J. H., and Kim, S. B., 2013, “A Clustering Algorithm-based Control Chart for Inhomogeneously Distributed TFT-LCD Processes." International Journal of Production Research, 51 (18), 5644-5657.

12. Kang, J. H., Lee, K. J., Son, J. H., Hyun, S. S., Kim, H. K., Hwang, G. D., and Kim, S. B., 2012, "Bootstrap-Based Multivariate Control Charts in Thin Film Transistor Liquid Crystal Display Manufacturing Processes.” Advanced Science Letters, 13(1), 579-583.

13. Kano M, Tanaka S, Hasebe S, Hashimoto I, and Ohno H., 2003, "Monitoring independent components for fault detection.” AIChE Journal. 49: 969-976.

14. Kim, S. B., Jitpitaklert, W., Park, S. K., and Hwang, S. J., 2012, "Data mining modelbased control charts for multivariate and autocorrelated processes." Expert Systems With Applications, 39(2), 2073-2081.

15. Kim, S.B., Sukchotrat, T., Park, S. K., 2011, “A nonparametric fault isolation approach through one-class classification algorithms." IIE Transactions, 43, 505-517.

16. Kourti, T., Nomikos, P., and MacGregor, J. F., 1995, "Analysis, monitoring and fault diagnosis of batch processes using multiblock and multiway PLS.” Journal of Process Control, 5(4), 277-284.

17. Lee, J.M., Yoo, C.K., Lee, I.B., 2004, "Statistical process monitoring with independent component analysis.” Journal of Process Control, 14: 467-485.

18. Li, W., Yue, H. H., Valle-Cervantes, S., and Qin, S. J., 2000, "Recursive PCA for adaptive process monitoring." Journal of Process Control, 10, 471-486. 
19. Liu, R. Y., and Singh, K., 1993, "A quality index based on data depth and multivariate rank tests.” Journal of the American Statistical Association, 88(421), 252-260.

20. Mason, R. L., Champ, C. W., Tracy, N. D., Wierda, S. J., and Young, J. C. 1997, “Assessment of multivariate process control techniques.” Journal of Quality Technology, 29(2), 140-143.

21. Montgomery, D. C., 2005, Introduction to Statistical Quality Control. 5th ed. New York: Wiley.

22. Phaladiganon, P., Kim, S. B., Chen, V. C., Jiang, W. 2013, "Principal component analysis-based control charts for multivariate nonnormal distributions" Expert Systems With Applications, 40, 3044-3054.

23. Phaladiganon, P., Kim, S. B., Chen, V. C., Baek, J. G., and Park, S. K. 2011, "Bootstrapbased $\mathrm{T}^{2}$ Multivariate Control Charts." Communications in Statistics-Simulation and Computation, 40 (5), 645-662.

24. Qin, S. J., 1998, "Recursive PLS algorithms for adaptive data monitoring." Computers and Chemical Engineering, 22, 503-514.

25. Runger, G. C. 1996, "Multivariate Statistical Process Control for Autocorrelated Processes.” International Journal of Production Research, 34, 1715-1724.

26. Shewhart, W. A., 1939, Statistical Method from the Viewpoint of Quality Control. ISBN 0-486-65232-7.

27. Sukchotrat, T., Kim, S. B., and Tsung, F. 2010, “One-class Classification-based Control Charts for Multivariate Process Monitoring." IIE Transactions, 42, 107-120. 
28. Sukchotrat, T., Kim, S.B., Tsui, K.L, and Chen, V.C.P., 2011. "Integration of classification algorithms and control chart techniques for monitoring multivariate processes.” Journal of Statistical Computation and Simulation, 81(12), 1897-1911.

29. Sun, R. and Tsung, F., 2003, "A kernel-distance-based multivariate control chart using support vector methods.” International Journal of Production Research, 41, 2975-2989.

30. Svensén, M., and Bishop, C. M., 2005, "Robust Bayesian mixture modelling." Neurocomputing, 64, 235-252.

31. Tax, D. M. J., 2001, “One-Class Classification.” Thesis, Delft University of Technology.

32. Tuerhong, G., Kim, S. B., Kang, P., Cho, S., 2014, "Hybrid novelty score-based multivariate control charts." Communications in Statistics - Simulation and Computation, $43,115-131$.

33. Wang, X., Kruger, U., and Irwin, G. W, 2005. "Process monitoring approach using fast moving window PCA.” Industrial \& Engineering Chemistry Research, 44(15), 56915702.

34. Woodall, W. H., 2000, "Controversies and Contradictions in Statistical Process Control." Journal of Quality Technology, 32 (4), 341-350.

35. Xie, X., and Shi, H., 2012, "Dynamic multimode process modeling and monitoring using adaptive Gaussian mixture models," Industrial \& Engineering Chemistry Research, 51(15), 5497-5505.

36. Yu, J., 2012, "A particle filter driven dynamic Gaussian mixture model approach for complex process monitoring and fault diagnosis." Journal of Process Control, 22(4), 778-788. 
37. Yu, J., and Qin, S. J., 2008, "Multimode process monitoring with Bayesian inferencebased finite Gaussian mixture models.” AIChE Journal, 54(7), 1811-1829.

38. Zhang, Y., An, J., and Zhang, H., 2013, “Monitoring of time-varying processes using kernel independent component analysis." Chemical Engineering Science, 88, 23-32.

39. Zhang, R., and Rudnicky, A. I., 2002, "A large scale clustering scheme for kernel kmeans. In Pattern Recognition," 2002. Proceedings. 16th International Conference on IEEE 4, 289-292. 